[0212-7199(2003) 20: 1; pp 37-45] ANALES DE MEDICINA INTERNA Copyright $\odot 2003$ ARAN EDICIONES, S.L.

An. Med InTERna (Madrid) Vol. 20, N. ${ }^{\circ} 1$, pp. 37-45, 2003

\section{Optimización en el tratamiento de formas complicadas de la enfermedad inflamatoria intestinal. Enfermedad de Crohn fistulizante}

\author{
M. PEÑATE, M. CRUZ-SANTAMARÍA², J. L. MENDOZA², A. S. PEÑA', \\ M. DÍAZ-RUBIO², J. GARCÍA-PAREDES ${ }^{2}$
}

\author{
Servicio de Gastroenterología. Hospital Universitario Insular de Gran Canaria. Las \\ Palmas de Gran Canaria. 'Departamento de Gastroenterología y Laboratorio de \\ Inmunogenética. Vrije Universiteit Medical Centre. Amsterdam. Holanda. \\ ${ }^{2}$ Unidad de Enfermedad Inflamatoria Intestinal. Servicio de Aparato Digestivo. Hospital \\ Clínico San Carlos. Madrid
}

OPTIMIZATION IN THE MANAGEMENT OF COMPLICATED FORMS OF THE INFLAMMATORY BOWEL DISEASE. FISTULAS IN CROHN'S DISEASE

Peñate M, Cruz-Santamaría M, Mendoza JL, Peña AS, Díaz-Rubio M, García-Paredes J. Optimización en el tratamiento de formas complica das de la enfermedad inflamatoria intestinal. Enfermedad de Crohn fistulizante. An Med Interna (Madrid) 2003; 20 : 37-45.

\section{INTRODUCCIÓN}

La enfermedad inflamatoria intestinal (EII) es un proceso crónico que puede afectar al colon (colitis ulcerosa) o a cualquier parte del aparato gastrointestinal (enfermedad de Crohn) y no tiene un tratamiento curativo en la actualidad. En su evolución natural se intercalan episodios de reactivación sintomáticos con periodos de inactividad. Su curso crónico recidivante se debe a la pérdida de la tolerancia, funcionamiento anómalo o a una curación defectuosa en la barrera mucosa. Los diferentes tratamientos intentan: obtener la remisión clínica en el brote agudo, mantenerla posteriormente el máximo tiempo posible con los menores efectos secundarios, conservar un estado nutricional adecuado y mejorar la calidad de vida de los pacientes.

El mejor conocimiento de los mecanismos por los que se produce la inflamación y los efectos secundarios asociados al uso continuado de los corticoides, que además no son útiles en el mantenimiento de la remisión, han llevado a la búsqueda de nuevos tratamientos para aplicarlos a grupos de pacientes concretos. En el tratamiento farmacológico sintético de la EII las aportaciones más importantes son: modificaciones químicas que aumentan la efectividad disminuyendo los efectos secundarios (la evolución desde la hidrocortisona a la budesonida y desde la sulfazalacina a los diferentes 5-aminosalicilatos) y la mejora en los parámetros farmacocinéticos (como ocurre con la azatioprina y la 6-mercaptopurina). Mediante la determinación de la actividad de la tiopurinmetiltransferasa (TPMT) podemos mejorar la efectividad y disminuir los efectos secundarios de la azatioprina y de la 6-mercaptopurina. A pesar de estos avances, una de las principales razones para continuar con el desarrollo de nuevos tratamientos es que los actuales necesitan semanas para ser efectivos y cuando lo consiguen, lo son sólo parcialmente. La mayoría de las veces, sobre todo en la enfermedad de Crohn (EC), no hay remisión histopatológica y en general no se previene la cirugía a largo plazo ni la degeneración maligna.

Todos estos factores, junto con la importancia de los mecanismos inmunológicos que regulan la inflamación, han hecho que se desarrollen agentes biológicos en el tratamiento. Estos nuevos fármacos tratan de forma simultánea de disminuir los niveles de antígenos bacterianos intraluminales y/o bloquear de forma selectiva la respuesta inmune dañina en la mucosa, como los anticuerpos monoclonales anti-TNF o los nuevos tratamientos que inhiben la respuesta Th1. El uso de estos tratamientos biológicos es más costoso $\mathrm{y}$, al igual que los tratamientos tradicionales, tampoco son curativos. Sus complicaciones a largo plazo aún están por ver y no sólo se trata de inducir la remisión sino de mantenerla. Además, en general, han sido más eficaces en la EC, concretamente en la forma fistulizante, descrita por primera vez en 1932 por Crohn y cols., que es la que tiene una mayor morbilidad.

Por tanto, debido a que los avances más importantes en el tratamiento farmacológico de la EII han sido en la EC fistulizante, aunque todos ellos son aplicables en alguna medida o momento a las restantes formas complicadas de la enfermedad, como son la corticodependencia o la corticoresistencia, es en la primera de estas complicaciones donde nos centraremos, para ver si realmente se ha optimizado el tratamiento con los fármacos "tradicionales" antes de emplear los nuevos.

Trabajo aceptado: 14 de mayo de 2002

Correspondencia: J. García-Paredes. Servicio Aparato Digestivo. Hospital Clínico San Carlos. C/ Martín Lagos s/n. 28040 Madrid. e-mail. jgarciap.hcsc@salud.madrid.org 


\section{ANTIBIÓTICOS}

\section{METRONIDAZOL}

En el tratamiento de la EC fistulizante sólo se ha valorado el tratamiento con metronidazol mediante estudios no controlados. En 1975 Ursing y Kamme obtuvieron el cierre de fístulas perianales en pacientes con EC tras el tratamiento con metronidazol (1). Posteriormente en 1980 Bernstein y cols. (2) con una dosis de $20 \mathrm{mg} / \mathrm{kg}$ de peso al día consiguieron una respuesta clínica en un $95 \%$ de los pacientes y una curación completa en un 56\% si la medicación se mantenía. La mejoría sintomática ocurrió en un $90 \%$ de los pacientes en 2 semanas y el $10 \%$ restante notó mejoría en 6-8 semanas. Brandt y cols. en 1982 (3) observaron una curación rápida de la enfermedad perineal con la misma dosis de $20 \mathrm{mg} / \mathrm{kg} /$ día, pero con una recurrencia sintomática en un $78 \%$ de los pacientes tras 4 meses de la suspensión. Jakobovits y Schuster en 1984 (4) comunicaron un $50 \%$ de curación de las fístulas con este tratamiento. En un estudio europeo al año siguiente (5) se comunicó una eficacia similar. En general, se produjo un cierre de las fístulas activas en el $40 \%$ de todos los pacientes tratados y en un $20 \%$ una disminución del drenaje.

Por tanto, puede concluirse que los ensayos no aleatorizados revelan que, como mínimo, la mitad de los pacientes con enfermedad perineal presentan una curación/mejoría con el metronidazol, pero que al discontinuarlo las fístulas recidivan, por lo que se precisaría un tratamiento de mantenimiento. Así mismo se necesita la realización de estudios controlados.

\section{CIPROFLOXACINO}

A principios de 1990 , debido a la alta incidencia de los efectos secundarios del metronidazol, se comienza a emplear el ciprofloxacino como tratamiento empírico en la EC. Aunque sigue siendo muy utilizado en la actualidad, su eficacia no ha sido suficientemente contrastada en el tratamiento de la EC fistulizante y cuando se ha hecho ha sido en estudios no controlados.

Turunen y cols. (6) emplearon en 1989 el ciprofloxacino en 8 pacientes con enfermedad perianal activa refractaria al metronidazol y a varios procedimientos quirúrgicos y en un paciente con una fístula enterocutánea a una dosis de 1.000 a $1.500 \mathrm{mg} /$ día durante 3-12 meses. Aunque los investigadores comunicaron la aparición de mejorías en todos los casos después del tratamiento, en 4 pacientes persistieron las alteraciones perianales y fue necesaria la realización de un tratamiento quirúrgico. Posteriormente nuevamente Turunen y cols. vieron que en 10 pacientes con EC perianal o fistulosa grave refractaria al tratamiento convencional, el fármaco era eficaz para curar las lesiones perianales y reducir las exacerbaciones, además de existir una baja incidencia de efectos secundarios (7).

Como con el metronidazol, las fístulas frecuentemente recurren una vez que el tratamiento se discontinúa y se necesita la realización de estudios controlados.

\section{METRONIDAZOL Y CIPROFLOXACINO}

Debido a la eficacia del metronidazol contra los gérmenes anaerobios y del ciprofloxacino contra los Gram-negativos ambos antibióticos han sido utilizados de manera combinada. En 1993 Solomon y cols. (8) comunicaron los resultados de un estudio retrospectivo de 14 pacientes con EC en el que 9 tenían fístulas complejas y 1 una fístula rectovaginal. Después de 12 semanas de tratamiento médico con metronidazol a una dosis de 500-1.500 mg al día y ciprofloxacino a una dosis de $1.000-1.500 \mathrm{mg}$ al día, hubo mejoría en 9 pacientes y curación en 3 , mientras que en un paciente no hubo cambios y otro empeoró, requiriendo una desviación proximal. Al interrumpir la medicación recurrieron los síntomas, necesitando repetir el tratamiento 9 de los 14 pacientes.

Se trata de un único estudio con ambos antibióticos, retrospectivo y en el que el tratamiento de las fístulas no fue el objetivo principal. Se necesitan más estudios, prospectivos, controlados y con el objetivo primario de ver respuesta en la enfermedad fistulosa.

\section{INMUNOSUPRESORES}

\section{AZATIOPRINA Y 6-MERCAPTOPURINA}

En general, la medicación inmunomoduladora tiene una excelente eficacia en el tratamiento de la EC fistulizante. Los fármacos más estudiados son la azatioprina y su metabolito, la 6-mercaptopurina. Estos dos fármacos no son intercambiables porque no hay estudios controlados que los comparen y porque la 6-mercaptopurina no es enzimáticamente convertible en azatioprina.

No se han realizado estudios controlados con azatioprina o 6-mercaptopurina en los que el principal objetivo sea el cierre de las fístulas en la EC perineal. Un metaanálisis llevado a cabo en 1995 (9) de cinco estudios aleatorizados y controlados con azatioprina y 6-mercaptopurina para la EC en el tratamiento del cierre de las fístulas (10-14) concluyó que 22 de 41 (54\%) pacientes tratados con azatioprina o 6-mercaptopurina respondieron, frente a 6 de $29(21 \%)$ de los tratados con placebo; el odd-ratio de curación de las fístulas fue de 4,44. Pero estos estudios tienen que ser interpretados con precaución porque el objetivo principal fue tratar los síntomas de la EC activa, más que el de las fístulas perineales.

Un estudio retrospectivo en 1985 (15) en el que se administró 6-mercaptopurina a 148 pacientes que no habían respondido a esteroides o a otras medicaciones, concluyó que en el 65\% (32/49) de los pacientes existía curación o mejoraban las fístulas internas y externas y en el $87 \%$ se presentaba curación o mejoría con la desaparición del dolor, sensibilidad anormal al tacto y las fístulas perirectales y abscesos.

El único ensayo aleatorizado y controlado con placebo (10) que evaluaba la eficacia de la 6-mercaptopurina en la EC obtuvo resultados similares. Se trataba de un estudio cruzado y a doble ciego basado en la administración de 6-mercaptopurina durante un año y de placebo durante otro. Aproximadamente el $25 \%$ de las fístulas cerraron mientras que el $50 \%$ restante mostró alguna mejoría.

\section{METOTREXATO}

El metotrexato (MTX) es un fármaco antagonista del ácido fólico con propiedades de antimetabolito y antiinflamato- 
rias. En los primeros estudios realizados para valorar su eficacia en la inducción de la remisión en los pacientes con EC no se informó de su efecto sobre las fístulas. En 1997 Muhadevan y cols. evaluaron su eficacia sobre 33 pacientes tratados de manera no controlada mediante una administración intramuscular. La remisión clínica se obtuvo en un $62 \%$ de los casos, entre ellos había 16 pacientes de los 33 con fístulas. De este grupo, con tratamiento 4 cerraron sus fístulas y 5 habían mejorado, proporcionando una respuesta de un $56 \%$. Cuando la dosis de medicación fue disminuida o pasada a medicación oral, se observaron frecuentes recidivas.

Otro estudio retrospectivo se realizó en 1999 por Vandeputte y cols. (16) para el tratamiento de 20 pacientes con EC refractaria, 8 de los cuales tenían enfermedad fistulizante. Los autores comunicaron que el MTX parenteral fue efectivo en la inducción de la remisión en un $70 \%$ de los pacientes intolerantes o resistentes a la azatioprina. Pero los autores no categorizaron las respuestas según el tipo de enfermedad; específicamente no comentan el número de pacientes con enfermedad fistulizante que obtuvieron remisión. Por tanto sería necesaria la realización de más ensayos clínicos para valorar su eficacia en la enfermedad fistulizante.

\section{CICLOSPORINA}

No se han publicado estudios randomizados y controlados designados específicamente para evaluar la eficacia de la ciclosporina en el cierre de las fístulas en los pacientes con EC. Existen ocho estudios no controlados con ciclosporina intravenosa para el tratamiento de la EC fistulizante que no ha respondido a los corticoides, metronidazol o a los antimetabolitos (17-24). La respuesta inicial completa fue de un $83 \%$. La ciclosporina fue administrada en infusión continua durante 24 horas a una dosis de $4 \mathrm{mg} / \mathrm{kg}$ de peso. La mejoría clínica se produjo rápidamente dentro de la primera semana. A los pacientes que respondieron se pautó ciclosporina oral posteriormente. El porcentaje de recidiva fue relativamente alto cuando se discontinuó este tratamiento $(19,22,23,25)$.

Estos datos no controlados sugieren que la ciclosporina intravenosa es eficaz para la EC fistulizante como puente hacia el tratamiento de mantenimiento con AZA, 6-MP o MTX. La frecuencia de recidiva después del cese del tratamiento oral fue de un $82 \%$. En los individuos que suspendieron la ciclosporina oral después de un periodo corto de uso de otros inmunosupresores no hubo tiempo para que éstos hubiesen hecho efecto. Sin embargo, deberían realizarse más ensayos aleatorizados y controlados con placebo para valorar el uso de esta medicación en la EC fistulizante.

\section{TACROLIMUS (FK 506)}

Se trata de un hongo (Streptomyces) derivado de los macrólidos que es similar a la ciclosporina a causa de su habilidad para inhibir la transcripción de la IL-2 en los linfocitos T-helper y ha sido efectivo para la prevención del rechazo tras un trasplante hepático. Los estudios realizados para valorar su efectividad en el Crohn con clínica fistulizante entre otras manifestaciones, son no controlados (26-28). Un estudio retrospectivo (26) que revisa las historias clínicas de los pacientes con EC fistulizante de la clínica Mayo tratados con tacrolimus en el periodo 1996-1998 incluye 11 pacientes con una dosis inicial oral de 0,15 a $0,31 \mathrm{mg} / \mathrm{kg}$ de peso. La totalidad de los mismos experimentaron mejoría y 7 (64\%) habían completado el cierre de las fístulas. El tratamiento fue continuado una media de 22 semanas como puente para la terapia de mantenimiento con azatioprina o 6-mercaptopurina. Un $73 \%$ de los pacientes habían mejorado sustancialmente después de que se discontinuara el tratamiento.

Se han descrito casos de pacientes con EC fistulizante resistentes a los tratamientos convencionales que han tenido una buena respuesta en dos meses permitiendo disminuir la dosis de corticoides o bien continuar con un tratamiento de mantenimiento posterior con inmunosupresores (29). Otros autores han planteado su utilización tópica al comprobarse su utilidad en trastornos cutáneos resistentes a ciclosporina; al ser "captado" por las cubiertas inflamadas se reduce la producción de citoquinas por parte de los queratinocitos. Se ha llevado a cabo un ensayo abierto (30) en 8 niños, 6 de ellos con EC (EC) perineal logrando la formulación tópica a dosis bajas $(0,5 \mathrm{mg} / \mathrm{g})$ a partir de la oral o intravenosa y mezclándolas con excipientes apropiados. Se comprobó una importante mejoría en 5 de estos niños en 6 semanas sin evidenciarse una absorción sistémica significativa. Sin embargo, puede producirse un empeoramiento claro de la clínica en caso de retirada o disminución rápida de la dosis del fármaco. Ierardi y cols. (31) plantean otro estudio abierto y no controlado en 13 pacientes con EC resistente a esteroides; 6 con fístulas, obteniendo buena respuesta (cierre completo de las mismas) en la mitad de ellos a dosis de $0,1-0,2 \mathrm{mg} / \mathrm{kg} /$ día asociado a mesalazina y logrando instaurar una pauta descendente de corticoides. En dos series de casos clínicos se comenta su efectividad en la EC con manifestación fistulizante entre otras, obteniéndose una rápida mejoría clínica y utilizándose también como tratamiento puente para otros inmunosupresores $(27,29)$.

Por tanto, la combinación del tacrolimus oral y la AZA o la 6-MP puede ser efectiva en el tratamiento de la EC con fístulas perianales. Deberían continuar los estudios controlados que evalúen su eficacia en este tipo de manifestación de la enfermedad.

\section{MICOFENOLATO MOFETIL}

Se trata de otro potente inmunosupresor que inhibe la proliferación de linfocitos bloqueando de forma selectiva la síntesis del nucleótido guanosina en las células T. Ha sido usado de forma efectiva para el tratamiento de la EC corticorefractaria en un estudio prospectivo, randomizado y controlado (32). También hay un estudio no controlado de pacientes con EC fistulizante (33) y estudios que sugieren que puede ser utilizado en pacientes con intolerancia a la azatioprina, de 12 pacientes estudiados 7 tenían una EC, fueron tratados con una dosis de $500 \mathrm{mg}$ b.i.d. durante una media de 12,5 semanas. 4 pacientes respondieron con mejoría sintomática, reducción de corticoides y mesalazina. Se necesitan por tanto, estudios prospectivos con este fármaco en la EC fistulizante.

\section{AGENTES BIOLÓGICOS}

El conocimiento de la patogénesis de la inflamación en la EII ha contribuido al desarrollo de fármacos de diseño, cuya 
función consiste en bloquear los efectos biológicos de una molécula diana mediante la inhibición de su síntesis endógena o bloqueando su acción biológica. En los últimos años, el TNF $\alpha$ ha sido el objetivo principal de estas estrategias, habiéndose desarrollado moléculas que interfieren con su efecto biológico mediante bloqueo de su producción o secreción (pentoxifilina, talidomida, bloqueantes de la enzima convertidora del TNF $\alpha$-TACE- y oligonucleótidos antisentido), anticuerpos monoclonales: un anticuerpo quimérico monoclonal anti-factor de necrosis tumoral alfa (Infliximab; cA2), otro anticuerpo humanizado monoclonal anti-TNF (CDP571) y un receptor TNF humano soluble recombinante unido a una proteína que neutralizan su unión al receptor (onercept, etanercept) $(34,35)$.

\section{INFLIXIMAB}

Recientemente se ha creado un anticuerpo monoclonal quimérico dirigido contra el TNF-a humano, el infliximab, formalmente conocido como cA2 (Centocor Inc., Malvern, PA). Es un anticuerpo monoclonal quimérico IgGk murino y humano (36-39) formado por la unión de las regiones variables de un anticuerpo monoclonal de ratón para el TNF humano (A2) con las regiones constantes de una inmunoglobulina humana IgG1k. Así el anticuerpo quimérico resultante presenta una menor inmunogenicidad. El mecanismo de acción se consigue gracias a la unión de alta afinidad del infliximab a las formas solubles y transmembrana del TNF-a bloqueando la interacción con sus receptores y neutralizando de esta forma su actividad biológica (40).

Se han publicado diferentes trabajos que demuestran la eficacia y seguridad del tratamiento con infliximab en pacientes con EII activa moderada-severa (intraluminal) y en EC fistulizante (41-51).
La eficacia del infliximab en la EC fistulizante que no responde al tratamiento convencional durante tres meses ha sido demostrada en un ensayo clínico (41) en 1999. Se realizó un estudio placebo-control con infliximab en 94 pacientes con EC fistulizante, de los cuales un $90 \%$ tenían fístulas perianales y un $10 \%$ fístulas abdominales activas, de al menos 3 meses de evolución (41). Los pacientes recibieron una de las tres pautas de tratamiento: placebo intravenoso ( 31 pacientes), $5 \mathrm{mg} / \mathrm{kg}$ intravenoso (31 pacientes) o $10 \mathrm{mg} / \mathrm{kg}$ intravenoso (32 pacientes). Todos los tratamientos fueron administrados en las semanas 0, 2 y 6 . El objetivo era obtener uno de los tres resultados siguientes: un descenso de un $50 \%$ o más de la línea de base en el número de fístulas abiertas que drenan durante al menos 2 visitas (tiempo total de un mes), que fue el objetivo principal; el cierre de todas las fístulas abiertas en al menos 2 visitas (tiempo total de un mes), que fue el objetivo secundario; y ausencia de respuesta. Un $68 \%$ de los pacientes que recibieron infliximab a una dosis de $5 \mathrm{mg} / \mathrm{kg}$ y un $56 \%$ de los que lo recibieron a una dosis de $10 \mathrm{mg} / \mathrm{kg}$ consiguieron el objetivo principal comparado con el $26 \%$ en el grupo placebo $(\mathrm{p}=0,002$ y $\mathrm{p}=0,020)$. También un $55 \%$ de los pacientes que recibieron $5 \mathrm{mg} / \mathrm{kg}$ y un $38 \%$ de los pacientes que recibieron $10 \mathrm{mg} / \mathrm{kg}$ habían cerrado todas las fístulas comparado con un $13 \%$ de los pacientes que recibieron placebo $(\mathrm{p}=0,001$ y $\mathrm{p}=$ 0,040 , respectivamente). Así se podía concluir que el infliximab era eficaz en la EC fistulizante, y la respuesta no era dosis dependiente. Estos resultados iniciales se han mantenido en la práctica clínica $(42,44-49)$ y además se ha demostrado la seguridad de las infusiones repetidas de infliximab como tratamiento de mantenimiento (Tabla I).

Recientemente se han presentado los resultados del ACCENT II (52), un estudio multicéntrico, aleatorizado y a doble ciego que valora el tratamiento a largo plazo de la EC fistulizante con infliximab. Se comparó la efectividad de dosis repetidas frente a un único ciclo de 3 infusiones en el manteni-

TABLA I

RESULTADOS DE LA EXPERIENCIA CLÍNICA CON INFLIXIMAB

Autor
Total pacientes Inflamatoria/Fistulosa/Ambas
Respuesta

Inflamatoria (respuesta/ remisión) Fistulosa (respuesta/remisión)
Cohen et al 2000 (42)

Farrell et al 2000 (43)

Ricart et al 2000 (44)

Fefferman et al 2001 (45)

Vermeire et al 2001 (46)

Hommes et al 2002 (47)

García-Paredes et al 2002 (48)
129$$
100
$$$$
57 / 33 /
$$

100

$61 / 26 / 13$

200

$12 / 72 / *$

233

73

$57 / 16$

52

$6 / 26 / 20$
I $(65 \% / 31 \%)$

$\mathrm{F}(78 \% / 24 \%)$

I $(60 \% /)$

$\mathrm{F}(69 \% /)$

I $(72 \% / 53 \%)$

$\mathrm{F}(66 \% / 31 \%)$

I $(70 \% /)$

$\mathrm{F}(84 \% /)$

$(73 \% /)$

I $(81 \% /)$

$\mathrm{F}(87 \% /)$

I $(83 \% / 66 \%)$

$\mathrm{F}(67 \% / 44.2 \%)$

* 14 pacientes por corticodependencia y 1 afectación de esófago 
miento del cierre de las fístulas. Se trataron 302 pacientes con una dosis de $5 \mathrm{mg} / \mathrm{kg}$ en las semanas 0,2 y 6 , respondiendo $195(69 \%)$ y no haciéndolo $83(31 \%)$. El grupo de 195 pacientes respondedores fue aleatorizado para recibir tratamiento con inyecciones repetidas cada 8 semanas a dosis de $5 \mathrm{mg} / \mathrm{kg}$ o bien placebo. La mediana del tiempo hasta la pérdida de la respuesta fue significativamente superior en el grupo tratado con inyecciones repetidas (> 40 semanas) que en el grupo placebo (14 semanas). El porcentaje de pacientes con respuesta completa a la semana 54 fue superior en el grupo tratado con infusiones repetidas (36\%) así como el descenso en el CDAI y la mejora en el índice de calidad de vida (IBDQ) con respecto al grupo placebo. Este estudio demuestra que la administración repetida cada 8 semanas de infliximab a dosis de $5 \mathrm{mg} / \mathrm{kg}$ es eficaz para mantener la respuesta clínica en la EC fistulizante.

El $11 \%$ de los pacientes tratados con infliximab desarrollaron abscesos perianales en el estudio de Present y cols. (41), posiblemente por el cierre cutáneo de los tractos fistulosos antes del cierre del propio tracto en sí, pero en la práctica clínica este porcentaje disminuye hasta el 5\% (44) cuando se combina la cirugía perianal, los antibióticos, los inmunosupresores y el infliximab, disminuyendo la incidencia de nuevos abscesos y podría ser un factor predictivo de respuesta (49).

Con respecto a posibles complicaciones, se han producido casos de tuberculosis activa tras el comienzo del tratamiento. Concretamente en España se han comunicado 10 casos (53, 54) en pacientes tratados con infliximab hasta Mayo del 2001. Por esta razón, se insiste en la realización de un despistaje previo a las infusiones (53). La GETECCU ha desarrollado un protocolo de actuación para determinar el riesgo en los pacientes que van a ser tratados (55) (Tabla II).

Por el importante impacto económico que supone la utilización del infliximab se han buscado factores predictivos de respuesta (56) que permitan un uso más racional de este fármaco. No se ha encontrado ninguna relación con la severidad y duración de la enfermedad, tratamientos previos (45) y polimorfismos del TNF, receptores del TNF y del gen NOD2 (CARD 15). Aunque es probable que bajos niveles séricos postinfusión de TNF y el uso concomitante con inmunosupresores sean factores predictivos independientes de respuesta al tratamiento. Otros factores predictivos de respuesta que se están estudiando y que es posible que tengan importancia son la localización de la enfermedad (colónica), la edad (46), la no respuesta a la primera infusión $(42,44,49)$ y el status ASCA y pANCA (57).

Recientemente se ha publicado un estudio coste-utilidad con el tratamiento médico inicial para las fístulas perianales en la EC donde mediante el modelo de Markov se simulaba un año de tratamiento comparando 6-mercaptopurina (6MP) y metronidazol (met) con: 3 infusiones de infliximab más 6mercaptopurina y metronidazol como segunda línea de tratamiento (intervención I), infliximab con reinfusiones periódicas (intervención II) y 6-mercaptopurina con metronidazol más infliximab como segunda línea de tratamiento (intervención III). Todas las estrategias tuvieron una efectividad similar pero el infliximab fue mucho más caro que $6 \mathrm{MP} / \mathrm{met}$. Así concluían que el beneficio del infliximab para el tratamiento de las fístulas perianales en la EC en un periodo de un año no justificaba el alto coste pero que se necesitaban estudios prospectivos para comparar directamente $6 \mathrm{MP} /$ met e infliximab (58) y quizá lo más importante, valorar las mejoras en la calidad de vida de los pacientes. En cambio Wong y cols., en forma de abstra en 1999 comunican que el coste de la EC es tres veces mayor que la de la colitis ulcerosa (costes directos como, el precio del fármaco, intervenciones quirúrgicas y hospitalización; costes indirectos como bajas laborales entre otros y costes intangibles como la calidad de vida de los pacientes) y que sólo las intervenciones quirúrgicas y los costes de hospitalización eran el $80 \%$ de los gastos. Así mismo concluían que el infliximab disminuía un 60\% la cirugía en las formas fistulizantes y como ésta era la mitad de los gastos directos, que existía un importante ahorro en los costes disminuyendo las visitas a urgencias, a consulta externa, medicaciones, ingresos hospitalarios e intervenciones quirúrgicas.

A pesar de que el infliximab ha demostrado ser efectivo en el tratamiento de las fístulas en la EC al mejorar la calidad de vida de los pacientes y permitir un manejo más sencillo de la enfermedad fistulosa, este beneficio se produce a corto plazo, incluso se discute el cierre de los tractos fistulosos por ecoendoscopia tras tres infusiones de inducción (59). Por lo tanto todavía son necesarios más estudios que permitan determinar el papel de los tratamientos concomitantes, los factores predictivos de respuesta y el papel de la cirugía tras la introducción del infliximab.

CDP-571

Recientemente se ha creado un anticuerpo humanizado monoclonal quimérico contra el TNF- $\alpha$ llamado CDP-571 (Celltech, Slough, England) por unión de las regiones determinantes complementarias de un anticuerpo monoclonal de ratón anti- humano TNF- $\alpha$ contra IgG4 con cadenas ligeras Kappa. Contiene un una secuencia $95 \%$ humana y $5 \%$ murina. El uso de este anticuerpo administrado de forma intravenosa se ha demostrado eficaz en el tratamiento de pacientes con EC activa y también en pacientes con enfermedad fistulizante (60).

TABLA II

PROTO CO LO DE ACTUACIÓN PARA DETERM INAR EL RIESGO DE TUBERCULOSIS

\begin{tabular}{llllll}
\hline Grado & Exposición & PPD & Rx tórax & Diagnóstico & Tratamiento \\
\hline 0 & No & - & Normal & No & No \\
I & Sí & BCG & Normal & Probable No & No Vigilancia \\
II & Sí desconocida & + & Normal & TB latente & Profilaxis \\
III & Sí o desconocida & + ó & Activa & TB activa & Tratamiento \\
IV & Sí o desconocida & + & Inactiva & TB superada & Profilaxis \\
\hline
\end{tabular}


Se completaron dos estudios multicéntricos nacionales para evaluar la eficacia del CDP-571 en pacientes con EC. El primero fue realizado con 71 pacientes corticodependientes tratados durante 16 semanas con una dosis inicial de $20 \mathrm{mg} / \mathrm{kg}$ y una segunda dosis de $10 \mathrm{mg} / \mathrm{kg} 8$ semanas más tarde. Los corticoides se discontinuaron a la semana 10 . El otro estudio (35) evaluó 169 pacientes con EC de moderada a severa incluyendo muchos pacientes que no habían respondido adecuadamente a los tratamientos actuales existentes. Los pacientes fueron tratados con CDP-571 a unas dosis de 10 y $20 \mathrm{mg} / \mathrm{kg}$ o con placebo y evaluados a las 24 semanas. Se evaluó su eficacia en el cierre de las fístulas de una forma adicional en los pacientes de ambos estudios. Un 50\% de los individuos con fístulas y un $15 \%$ en el grupo placebo $(\mathrm{p}=0,074)$ tuvieron más de un $50 \%$ de cierre. Para poder valorar correctamente estos resultados tenemos que tener en cuenta que el objetivo principal del estudio fue valorar la respuesta clínica en la semana segunda como una disminución del índice de actividad de la EC igual o más de 70 puntos. La conclusión del estudio fue que el CDP-571 a una dosis inicial de 10 a 20 $\mathrm{mg} / \mathrm{kg}$ es seguro y efectivo para el tratamiento de los pacientes con EC moderada-severa.

Los resultados preliminares sugieren que el retratamiento con $10 \mathrm{mg} / \mathrm{kg}$ con un intervalo de 8-12 semanas puede ser beneficioso. Los efectos adversos fueron: reacciones de la infusión, reacciones de hipersensibilidad retardada, formación de anticuerpos humanos antiquiméricos, formación de anticuerpos antidoble hebra de DNA y en raros casos lupus inducido por fármacos $(36,61)$. Sin embargo, se necesitan estudios adicionales para valorar su eficacia en el tratamiento de la EC y en concreto en la enfermedad fistulizante.

\section{OTROS TRANTAMIENTOS ANTI-TNF- $\alpha$}

\section{Talidomida}

Originalmente fue comercializada como sedante y antiemético y aunque en 1961 tuvo que ser retirada del mercado al comprobarse su efecto teratogénico, diversos ensayos clínicos han demostrado su eficacia terapéutica en trastornos granulomatosos y otros procesos de base inmunitaria en los que el TNF- $\alpha$ se ha implicado como destacado mediador inflamatorio. La mejoría sintomática que se ha comunicado en forma de casos clínicos de pacientes con EC resistente a otros tratamientos, incluyendo inmunosupresores (62) y las propiedades anti-TNF del fármaco han permitido desarrollar hasta la fecha dos ensayos clínicos abiertos. El mecanismo exacto de acción todavía no se conoce por completo, pero se sabe que inhibe la producción y liberación de TNF- $\alpha$ e IL-12 así como la angiogénesis $(63,64)$. De los dos ensayos clínicos citados, el de Vasiliauskas y cols. (65) no está específicamente diseñado para evaluar su efectividad en la enfermedad fistulosa, pero de los 12 pacientes incluidos, 6 tenían fístulas perianales activas al inicio del estudio. 5 de ellos $(83 \%)$ tuvieron una mejoría importante de los síntomas relacionados (disminución del débito, induración, dolor, etc.) pudiendo completar las 12 semanas de tratamiento 4 de estos pacientes. El estudio de Ehrenpreis y cols. (66) sí valora específicamente el resultado en pacientes fistulosos mediante el GIS (Goal Interval Score) propuesto Present y cols. (10) que incluye 3 parámetros clínicos: disminución de la dosis de esteroides, cierre de las fístu- las y mejoría de otros síntomas y signos. De un total de 22 pacientes, 13 tienen fístulas perianales resistentes a otros tratamientos. 9 de ellos completaron 12 semanas de tratamiento y presentaron buena respuesta $(69 \%)$ con remisión clínica en el $46 \%$ de los casos; incluso en 2 de estos pacientes había fracasado previamente el tratamiento con infliximab.

Ambos estudios obtienen buenos resultados a dosis relativamente bajas (100-300 mg/día), permiten la retirada de los corticoides y ofrecen buena tolerancia. Aunque no hay que olvidar que son no controlados, por lo que se necesitan estudios controlados para determinar eficacia, dosis más adecuada y perfil de seguridad, además de pensar en la posibilidad de establecer modificaciones en su molécula que maximicen sus propiedades antiinflamatorias y minimicen su toxicidad potencial.

\section{Etanercept y onercept}

Para que el TNF- $\alpha$ desarrolle su efecto biológico es preciso que se una a receptores específicos de membrana (tipo I ó p55 y tipo II ó p75) constituidos por tres dominios: intracitoplasmático, transmembrana y extracelular. Éste último se escinde mediante acción proteolítica dando lugar a sus formas solubles (TBP-1 y 2; presentes en suero y orina entre otros fluidos) que, aunque mantienen su capacidad de unión al TNF- $\alpha$, participan en su neutralización de modo que muchos de sus efectos biológicos se ven bloqueados por estas proteínas de unión.

Se han desarrollado formas recombinantes de ambas formas solubles. El rh TBP-1 u onercept ha sido desarrollado por Serono en células de ovario de hámster chino. Hasta ahora se han realizado 3 ensayos en voluntarios sanos sin que se hayan descrito acontecimientos adversos graves o la presencia de anticuerpos frente a la molécula (67). Su perfil de seguridad y sus características farmacocinéticas hacen que sea un buen candidato para neutralizar / antagonizar los altos niveles de TNF- $\alpha$ que se presentan en diversos trastornos inflamatorios, inmunitarios y cardiovasculares. De hecho, se están desarrollando ensayos, algunos abiertos y otros aleatorizados y controlados frente a placebo, no solo en EC, sino también en artritis reumatoide o síndrome de isquemia-reperfusión.

Por lo que se refiere a la forma recombinante de la otra proteína de fusión (rh TBP-2 o etanercept), se han realizado hasta la fecha 2 ensayos en EC. D'Haens y cols. (68) lo emplean a dosis de $25 \mathrm{mg} /$ día sc, 2 veces a la semana durante 12 semanas en 10 pacientes con EC moderada a severa refractaria a tratamiento estándar, comprobando buena respuesta clínica, endoscópica y en menor medida histológica. Sandborn y cols. (69) emplean la misma pauta durante 8 semanas en 43 pacientes con EC de similares características, pero en esta ocasión el ensayo es aleatorizado y controlado frente a placebo. A las 4 semanas responde un $39 \%$ de los tratados con etanercept frente al $45 \%$ de los que recibieron placebo.

Ninguno de los 2 ensayos menciona si algún paciente presentaba enfermedad fistulizante y qué respuesta se obtuvo. Aunque el etanercept haya sido seguro, no ha sido efectivo, por lo que se plantea el empleo de dosis mayores o una mayor frecuencia de administración. Tanto onercept como etanercept suponen una nueva estrategia en el control de la EC, lo que justifica nuevas investigaciones; de hecho ya se están desarrollando ensayos controlados frente a placebo y de búsqueda de dosis. 


\section{LEUCOCITOAFÉRESIS}

A principios de la década de los 80 , empezaron a usarse diferentes técnicas de aféresis en algunas patologías de origen autoinmune, con la intención de eliminar selectivamente de la circulación células o componentes plasmáticos que pudieran estar implicados en su patogenia. En 1984 Bicks y cols. (70) realizan linfoplasmaféresis en 8 pacientes con EC activa severa; 5 de ellos responden, pero de forma poco predecible y no coste-efectiva. Diez años más tarde, Lerebours y cols (71) desarrollan u estudio aleatorizado y controlado frente a placebo en pacientes con EC en remisión tras tratamiento corticoideo, sin que demuestre utilidad en la prevención de recidivas precoces ( $83 \%$ en pacientes con linfocitoaféresis vs $62 \%$ en grupo control tras 18 semanas de seguimiento).

Ningún estudio menciona o evalúa de forma específica los resultados en pacientes fistulosos, pero la técnica, dado su efecto inmunosupresor, podría ser una buena alternativa al tratamiento farmacológico o bien coadyuvante (72); en cualquier caso se precisarán estudios bien diseñados para conocer su papel exacto.

\section{DIETA ELEMENTAL, REPOSO INTESTINAL Y NUTRICIÓN PARENTERAL}

El papel de la dieta en el desarrollo de la enfermedad también se ha estudiado como tratamiento primario y adyuvante en la EII. Se han demostrado beneficios con el uso de dieta elemental o polimérica en la enfermedad en fase aguda y durante el mantenimiento de la remisión.

En los pacientes con EII aparecen numerosas alteraciones nutricionales por lo que se ha visto la importancia de mejorar el estado nutricional general. La malnutrición calórico-proteica contribuye a una curación insuficiente de las heridas y fístulas y a un aumento de la morbilidad y mortalidad quirúrgicas. Existe un consenso respecto a hacer un esfuerzo por aumentar al máximo el estado alimentario de los pacientes con EII.

El uso de las dietas elementales y la nutrición parenteral en pacientes con EC y enfermedad perianal no han sido suficientemente estudiados (73-82). Calam y cols. (83) comunicaron sus resultados en 6 pacientes en los que se usó una dieta elemental para tratar fístulas perianales en la EC. En 4 casos $(66 \%)$ se produjo mejoría con el tratamiento con dieta elemental, pero las fístulas cerraron completamente sólo en un paciente $(17 \%)$. No hay estudios con la nutrición parenteral y la enfermedad perianal. El uso de dieta elemental o nutrición parenteral total como tratamiento primario de la EC perianal no es recomendada en principio ante la no existencia de evidencia concluyente.

\section{TECNOLOGÍA DEL DNA Y TRANSFERENCIA GENÉTICA}

Los avances recientes sugieren que el futuro en el estudio de la EII está en el uso de una clasificación basada en el genotipo. Se ha descubierto el gen NOD2 o CARD15 relacionado con la susceptibilidad a la EC. También el inhibidor de la señal TGF- $\beta_{1}$, llamado Smad7, está sobreexpresado en la mucosa de los pacientes y purificados en las células T mucosas. Además los oligonucleótidos específicos antisentido para el Smad7 reducen la expresión de la proteína $\operatorname{Smad7}$ en las células aisladas de los pacientes, permitiendo a las células responder al TGF- $\beta_{1}$. Por tanto, la tecnología del DNA y la transferencia genética probablemente superarán y serán más eficientes que la terapia biológica.

\section{MISCELÁNEA}

Se han utilizado otros y variados tratamientos en la EC fistulizante. Por ejemplo el oxígeno hiperbárico, que ha demostrado su eficacia en algunos casos $(84,85)$, pero estos estudios no eran prospectivos, ciegos, ni randomizados. El uso de una desviación fecal temporal ha sido utilizada ocasionalmente con éxito, aunque se observa frecuentemente la recidiva de la fístula en la zona de la ileostomía o colostomía (8688). La protectomía en los individuos con afectación perirectal severa también ha sido efectiva, a pesar de la mutilación que este proceder supone (89). Resultados preliminares con el uso del pegamento de fibrina (90) y el factor plasmático XIII concentrado (91) muestran la eficacia de estos en algunas fístulas perianales. Se necesitan más estudios antes de poder valorarlos adecuadamente y recomendar su uso de una forma rutinaria.

\section{DISCUSIÓN Y CONCLUSIONES}

Los principales objetivos en el tratamiento de la EII son; mejorar y/o mantener la calidad de vida de los pacientes, prevenir y tratar los síntomas, evitar los efectos adversos y disminuir las complicaciones asociadas. El tratamiento farmacológico ha avanzado mucho en los últimos años y los productos biológicos han despertado un gran interés, pero probablemente no se ha puesto suficiente énfasis en la descripción de sus fallos, limitaciones y complicaciones.

Dentro de la EII, el tratamiento de los pacientes con EC fistulizante es particularmente complejo y la elección entre una opción médica o quirúrgica depende siempre de múltiples factores, como la localización, severidad de los síntomas, número y complejidad de los tractos fistulosos, presencia o ausencia de enfermedad rectal, historia previa de cirugía local, estado nutricional del paciente, tiempo que tarda en recidivar, comorbilidad y estado físico asociados. Podríamos preguntarnos por qué no empezar primero con el tratamiento con antiTNF- $\alpha$ en casos concretos en los que sabemos que la enfermedad es más agresiva como ocurre en la enfermedad fistulizante. Es probable que utilizarlo de primera línea en este momento no sea lo más adecuado pero sí se podría administrar como tratamiento puente a los inmunosupresores en algunos casos. Quizá los problemas sean su seguridad a largo plazo, coste y el desarrollo de enfermedades neoplásicas y autoinmunes.

Para concluir, como hemos visto, sólo existe un estudio publicado controlado, prospectivo y randomizado para valorar la eficacia y seguridad del tratamiento en la EC fistulizante que es con infliximab, mostrando eficacia clínica sobre placebo de forma estadísticamente significativa. El resto de los fármacos utilizados en el tratamiento de la EC fistulizante se han estudiado de forma no controlada y en muchos casos han sido analizados de forma retrospectiva, sin utilizarse el tratamiento de la enfermedad fistulizante como objetivo primario. Se 
necesitan por tanto, estudios randomizados, controlados, prospectivos y multicéntricos para evaluar la eficacia de los fármacos "tradicionales" en la EC fistulizante. Así, usándolos de una forma más eficiente, obtendríamos mejores resultados y agotaríamos todas sus posibilidades terapéuticas antes de pautar los productos más recientes.

Cabe esperar que un mayor conocimiento de la cascada inflamatoria y del origen de la EII lleve a unos tratamientos más específicos y potentes. Sin embargo, una gran cantidad de pacientes responde sólo de una forma temporal o insufi- ciente a los fármacos disponibles o desarrollan efectos secundarios graves. Por ello, el futuro en el estudio de la EII está en el uso de una clasificación basada en el genotipo. La tecnología del DNA y la terapia genética probablemente superarán y serán más eficientes que la terapia biológica. Hasta que llegue ese momento, si se hacen realidad las esperanzas que han alimentado los nuevos productos biotecnológicos deberán seguir aumentando las investigaciones de laboratorio y clínicas acerca de todos los aspectos de la EII.

\section{Bibliografía}

1. Ursing B, Kamme C. Metronidazole for Crohn's disease. Lancet 1975; 1: 775-7.

2. Bernstein LH, et al. Healing of perineal Crohn's disease with metronidazole. Gastroenterology 1980; 79: 357-65.

3. Brandt LJ, et al. Metronidazole therapy for perineal Crohn's disease: a follow-up study. Gastroenterology 1982; 83: 383-7.

4. Jakobovits J, Schuster MM. Metronidazole therapy for Crohn's disease and associated fistulae. Am J Gastroenterol 1984; 79: 533-40.

5. Schneider MU, et al. Metronidazole in the treatment of Crohn disease. Results of a controlled randomized prospective study. Dtsch Med Wochenschr 1985; 110: 1724-30.

6. Turunen U, Farkkila M, Seppala K. Long-term treatment of perianal or fistulous Crohn's disease with Ciprofloxacin. Scand J Gastroenterol 1989; 24 (Supl. 148): 144.

7. Turunen U, Farkkila V, Valtonen V. Long-term outcome of ciprofloxacin treatment in severe perianal or fistulous Crohn's disease. Gastroenterology 1999; p. A793.

8. Solomon $\mathrm{M}$, et al. Combination ciprofloxacin and metronidazole in severe perianal Crohn's disease. Can J Gastroenterol 1993; 7: 571-573.

9. Pearson DC, et al. Azathioprine and 6-mercaptopurine in Crohn disease. A meta-analysis. Ann Intern Med 1995; 123: 132-42.

10. Present DH, et al. Treatment of Crohn's disease with 6-mercaptopurine. A long-term, randomized, double-blind study. N Engl J Med 1980; 302: 981-7.

11. Klein M, et al. Treatment of Crohn's disease with azathioprine: a controlled evaluation. Gastroenterology 1974; 66: 916-22.

12. Rosenberg JL, et al. A controlled trial of azathioprine in Crohn's disease. Am J Dig Dis 1975; 20: 721-6.

13. Rhodes J, et al. Controlled trial of azathioprine in Crohn's disease. Lancet 1971; 2: 1273-6.

14. Willoughby JM, et al. Controlled trial of azathioprine in Crohn's disease. Lancet 1971; 2: 944-7.

15. Korelitz BI and DH. Present, Favorable effect of 6-mercaptopurine on fistulae of Crohn's disease. Dig Dis Sci 1985; 30: 58-64.

16. Vandeputte L, et al. Methotrexate in refractory Crohn's disease. Inflamm Bowel Dis 1999; 5: 11-5.

17. Fukushima T, et al. Effects of cyclosporin A on active Crohn's disease. Gastroenterol Jpn 1989; 24: 12-5.

18. Lichtiger S. Cyclosporine therapy in inflammatory bowel disease: openlabel experience. Mt Sinai J Med 1990; 57: 315-9.

19. Hanauer SB, Smith MB. Rapid closure of Crohn's disease fistulas with continuous intravenous cyclosporin A. Am J Gastroenterol 1993; 88: 646-9

20. Present DH, Lichtiger S. Efficacy of cyclosporine in treatment of fistula of Crohn's disease. Dig Dis Sci 1994; 39: 374-80.

21. Markowitz J, et al. Long-term 6-mercaptopurine treatment in adolescents with Crohn's disease. Gastroenterology 1990; 99: 1347-51.

22. Hinterleitner TA, et al. Combination of cyclosporine, azathioprine and prednisolone for perianal fistulas in Crohn's disease. Z Gastroenterol 1997; 35: 603-8.

23. Egan LJ, Sandborn WJ, Tremaine WJ. Clinical outcome following treatment of refractory inflammatory and fistulizing Crohn's disease with intravenous cyclosporine. Am J Gastroenterol 1998; 93: 442-8.

24. Gurudu SR, et al. Cyclosporine therapy in inflammatory bowel disease:

short-term and long-term results. J Clin Gastroenterol 1999; 29: 151-4.

25. Sandborn WJ. Cyclosporine therapy for inflammatory bowel disease: definitive answers and remaining questions. Gastroenterology 1995; 109: 1001-3

26. Lowry PW, et al. Combination therapy with oral tacrolimus (FK506) and azathioprine or 6-mercaptopurine for treatment-refractory Crohn's disease perianal fistulae. Inflamm Bowel Dis 1999; 5: 239-45

27. Sandborn WJ, Preliminary report on the use of oral tacrolimus (FK506) in the treatment of complicated proximal small bowel and fistulizing Crohn's disease. Am J Gastroenterol 1997; 92: 876-9.

28. Fellermann K, et al. Steroid-unresponsive acute attacks of inflammatory bowel disease: immunomodulation by tacrolimus (FK506). Am J Gastroenterol 1998; 93: 1860-6.

29. Ierardi E, et al. Oral tacrolimus (FK 506) in Crohn's disease complicated by fistulae of the perineum. J Clin Gastroenterol 2000; 30: 200-2.

30. Casson DH, et al. Topical tacrolimus may be effective in the treatment of oral and perineal Crohn's disease. Gut 2000; 47: 436-40.

31. Ierardi E, et al. Long-term tacrolimus: a promising therapeutic approach for Crohn's disease. Transplant Proc 2001; 33: 2107-9.

32. Neurath MF, et al. Gut 1999; 44: 625-8.

33. Fickert P, et al. Mycophenolate mofetil in patients with Crohn's disease Am J Gastroenterol 1998; 93: 2529-32.

34. Van Assche G, Rutgeerts P. Anti-TNF agents in Crohn's disease. Expert Opin Investig Drugs 2000; 9: 103-11.

35. Sandborn WJ, et al. An engineered human antibody to TNF (CDP571) for active Crohn's disease: a randomized double-blind placebo-controlled trial. Gastroenterology 2001; 120: 1330-8.

36. Sandborn WJ, Hanauer SB. Antitumor necrosis factor therapy for inflammatory bowel disease: a review of agents, pharmacology, clinical results, and safety. Inflamm Bowel Dis 1999; 5: 119-33.

37. Murch SH, et al. Serum concentrations of tumour necrosis factor alpha in childhood chronic inflammatory bowel disease. Gut 1991; 32: 913-7.

38. Braegger CP, et al. Tumour necrosis factor alpha in stool as a marker of intestinal inflammation. Lancet 1992; 339: 89-91.

39. Murch SH, et al. Location of tumour necrosis factor alpha by immunohistochemistry in chronic inflammatory bowel disease. Gut 1993; 34: 1705-9.

40. Targan SR, et al. A short-term study of chimeric monoclonal antibody cA2 to tumor necrosis factor alpha for Crohn's disease. Crohn's Disease cA2 Study Group. N Eng1 J Med 1997; 337: 1029-35.

41. Present $\mathrm{DH}$, et al. Infliximab for the treatment of fistulas in patients with Crohn's disease. N Engl J Med 1999; 340: 1398-405.

42. Cohen RD, TsangJF, Hanauer SB. Infliximab in Crohn's disease: first anniversary clinical experience. Am J Gastroenterol 2000; 95: 3469-77.

43. Farrell RJ, et al. Clinical experience with infliximab therapy in 100 patients with Crohn's disease. Am J Gastroenterol 2000; 95: 3490-7.

44. Ricart E, et al. Infliximab for Crohn's disease in clinical practice at the Mayo Clinic: the first 100 patients. Am J Gastroenterol 2001; 96: 722-9.

45. Fefferman DLP, Reinert S, Alsahli M, Abdallah Hani MA, Falchuk K, Peppercorn M, Farrell R, Shah S. Smoking, age, duration of disease, gender, and other clinical factors do not predict response to infliximab in Crohn's disease patients. Gastroenterology 2001; 120 (Supl. I): 320.

46. Vermeire SLE, Carbonez A, Van Assche G, Noman M, Belaiche J, De Vos M, Van Gossum A, Pescatore P, Fiasse R, Pelckmans P, Reynaert H, 
D'Haens G, Rutgeerts P. Logistic regression of clinical parameters influencing response to infliximab. Gastroenterology 2001; 120 (Supl. I): 3149.

47. Hommes DW, et al. Infliximab treatment for Crohn's disease: one-year experience in a Dutch academic hospital. Inflamm Bowel Dis 2002; 8: 81-6.

48. García-Paredes J, González Lara V, Abreu L, Fernández Blanco I, Solis Herruzo JA, Mate J, López San Román A, Mendoza JL, Pérez Calle JL, Vera MI, Froilán C, Casis B, Sánchez F, Llorca J, Olivencia P. Experiencia clínica con infliximab en Enfermedad de Crohn. V Reunión Nacional de la Asociación Española de Gastroenterología en Madrid (Abstract), 2002.

49. Mendoza JL, García-Paredes J, Cruz Santamaria DM, Lana R, Ramírez Fernández E, Rodríguez Asteaga E, Díaz-Rubio M. Tratamiento con infliximab y factores predictivos de respuesta en la Enfermedad de Crohn. Rev Esp Enf Dig 2002; 94: 269-274.

50. Noman MBF, D'Haens G. HACA formation after infliximab (Remicade) treatment in Crohn disease is clearly associated with infusion reactions. Gastroenterology, 2001; 120 (Supl. I): 3150.

51. Farrell RJ, AM, Falchuk KR, Peppercorn MA, Michetti P. Human antichimeric antibody levels correlate with lack of response and infusion reactions following infliximab therapy. Gastroenterology 2001; 120 (Supl.): 364

52. Sands BE, et al. Long-term treatment of fistulizing Crohn's disease: response to infliximab in the ACCENT II trial through 54 weeks. Gastroenterology 2002; 122: A81-82.

53. Keane J, et al. Tuberculosis associated with infliximab, a tumor necrosis factor alpha-neutralizing agent. N Engl J Med 2001; 345: 1098-104.

54. Nunez Martínez $\mathrm{O}$, et al. Reactivation tuberculosis in a patient with anti-TNF-alpha treatment. Am J Gastroenterol 2001; 96: 1665-6.

55. GETECCU. Guía de consenso sobre tuberculosis y tratamiento de la enfermedad inflamatoria intestinal con infliximab. 2001 Junio.

56. Rugeerts P. A critical assessment of new therapies in inflammatory bowel disease. J Gastroenterol Hepatol 2002; 17 (Supl.): S177-S186.

57. Taylor KD, et al. ANCA pattern and LTA haplotype relationship to clinical responses to anti-TNF antibody treatment in Crohn's disease. Gastroenterology 2001; 120: 1347-55.

58. Arseneau KO, et al. Cost-utility of initial medical management for Crohn's disease perianal fistulae. Gastroenterology 2001; 120: 1640-56.

59. van Bodegraven AA, et al. Endosonographic evidence of persistence of Crohn's disease-associated fistulas after infliximab treatment, irrespective of clinical response. Dis Colon Rectum 2002; 45: 39-45; discussion 45-6.

60. Stack WA, et al. Randomised controlled trial of CDP571 antibody to tumour necrosis factor-alpha in Crohn's disease. Lancet 1997; 349: 521 4.

61. Hanauer SB. Review article: safety of infliximab in clinical trials. Aliment Pharmacol Ther 1999; 13 (Supl. 4): 16-22; discussion 38.

62. Odeka EB, Miller V. Thalidomide in oral Crohn's disease refractory to conventional medical treatment. J Pediatr Gastroenterol Nutr 1997; 25: 250-1.

63. Wettstein AR, Meagher AP. Thalidomide in Crohn's disease. Lancet 1997; 350: 1445-6.

64. Bousvaros A, Mueller B. Thalidomide in gastrointestinal disorders. Drugs 2001; 61: 777-87.

65. Vasiliauskas EA, et al. An open-label pilot study of low-dose thalidomide in chronically active, steroid-dependent Crohn's disease. Gastroenterology 1999; 117: 1278-87.

66. Ehrenpreis ED, et al. Thalidomide therapy for patients with refractory Crohn's disease: an open-label trial. Gastroenterology 1999; 117: 1271-7.

67. Trinchard-Lugan I, et al. Safety, pharmacokinetics and pharmacodyna- mics of recombinant human tumour necrosis factor-binding protein-1 (Onercept) injected by intravenous, intramuscular and subcutaneous routes into healthy volunteers. Eur Cytokine Netw 2001; 12: 391-8.

68. D'Haens $\mathrm{G}$, et al. Etanercept in the treatment of active refractory Crohn's disease: a single-center pilot trial. Am J Gastroenterol 2001; 96: 2564-8.

69. Sandborn WJ, et al. Etanercept for active Crohn's disease: a randomized, double-blind, placebo-controlled trial. Gastroenterology 2001; 121 : 1088-94.

70. Bicks RO, Groshart KD. The current status of T-lymphocyte apheresis (TLA) treatment of Crohn's disease. J Clin Gastroenterol 1989; 11: 136-

71. Lerebours E, et al. Treatment of Crohn's disease by lymphocyte apheresis: a randomized controlled trial. Groupe d'Etudes Therapeutiques des Affections Inflammatoires Digestives. Gastroenterology 1994; 107: 357-61.

72. Yamaji K, et al. Current therapeutic apheresis technologies for inflammatory bowel disease. Ther Apher 1998; 2: 105-8.

73. Voitk AJ, et al. Use of elemental diet during the adaptive stage of short gut syndrome. Gastroenterology 1973; 65: 419-26.

74. Segal W. Nutrition and family practice. Aust Fam Physician 1977; 6: 129-43.

75. Axelsson C, Jarnum S. Assessment of the therapeutic value of an elemental diet in chronic inflammatory bowel disease. Scand J Gastroenterol 1977; 12: 89-95.

76. Russell RI, Hall MJ. Elemental diet therapy in the management of complicated Crohn's disease. Scott Med J 1979; 24: 291-5.

77. Teahon $\mathrm{K}$, et al. Ten years' experience with an elemental diet in the management of Crohn's disease. Gut 1990; 31: 1133-7.

78. Jones VA. Comparison of total parenteral nutrition and elemental diet in induction of remission of Crohn's disease. Long-term maintenance of remission by personalized food exclusion diets. Dig Dis Sci 1987; 32; 12 (Supl.): 100S-107S

79. Fukuda $\mathrm{Y}$, et al. Efficacy of nutritional therapy for active Crohn's disease. J Gastroenterol 1995; 30 (Supl. 8): 83-7.

80. Milewski PJ, Irving MH. Parenteral nutrition in Crohn's disease. Dis Colon Rectum 1980; 23: 395-400.

81. Harford FJ Jr., Fazio VW. Total parenteral nutrition as primary therapy for inflammatory disease of the bowel. Dis Colon Rectum 1978; 21 : $555-7$.

82. Greenberg GR, et al. Controlled trial of bowel rest and nutritional support in the management of Crohn's disease. Gut 1988; 29: 1309-15.

83. Calam J, Crooks PE, Walker RJ. Elemental diets in the management of Crohn's perianal fistulae. JPEN J Parenter Enteral Nutr 1980; 4: 4-8.

84. Lavy A, et al. Hyperbaric oxygen for perianal Crohn's disease. J Clin Gastroenterol 1994; 19: 202-5.

85. Colombel JF, et al. Hyperbaric oxygenation in severe perineal Crohn's disease. Dis Colon Rectum 1995; 38: 609-14.

86. Grant DR, Cohen Z, McLeod RS. Loop ileostomy for anorectal Crohn's disease. Can J Surg 1986; 29: 32-5.

87. Zelas P, Jagelman DG. Loop illeostomy in the management of Crohn's colitis in the debilitated patient. Ann Surg 1980; 191: 164-8.

88. Harper PH, Kettlewell MG, Lee EC. The effect of split ileostomy on perianal Crohn's disease. Br J Surg 1982; 69: 608-10.

89. Farmer RG, Hawk WA, Turnbull RB Jr. Indications for surgery in Crohn's disease: analysis of 500 cases. Gastroenterology 1976; 71: 24550.

90. Abel ME, et al. Autologous fibrin glue in the treatment of rectovaginal and complex fistulas. Dis Colon Rectum 1993; 36: 447-9.

91. Oshitani N, et al. Treatment of Crohn's disease fistulas with coagulation factor XIII. Lancet 1996; 347: 119-20. 